\title{
ARTí́CULO
}

\section{Análisis de la comunidad de peces de descarte en la pesca de arrastre de camarón (temporada de lluvias 2013) en la zona centro-sur del litoral veracruzano, México}

Fish discard by-catch shrimp trawl (rainy season 2013) in south central coast of Veracruz, Mexico

\author{
Angel Morán-Silva1, Rafael Chávez-López ${ }^{2}$, Ma. de Lourdes Jiménez-Badillo ${ }^{1 *}$, Sergio \\ Cházaro-Olvera ${ }^{3}$, Gabriela Galindo-Cortes ${ }^{1}$ y Cesar Gabriel Meiners-Mandujano ${ }^{1}$
}

'Instituto de Ciencias Marinas y Pesquerías, Universidad Veracruzana, Cuerpo Académico de Manejo y Conservación de Recursos Acuáticos (CAMyCRA), Hidalgo 617, Col. Río Jamapa, Boca del Río, Veracruz, México C.P. 94290. *ljimenez@uv.mx

${ }^{2}$ Laboratorio de Ecología Estuarina, Facultad de Estudios Superiores Iztacala, Universidad Nacional Autónoma de México, Av. de los Barrios No. 1, Los Reyes Iztacala, Tlalnepantla, Estado de México, México C.P 54090

${ }^{3}$ Laboratorio de Crustáceos, Facultad de Estudios Superiores Iztacala, Universidad Nacional Autónoma de México, Av. de los Barrios No. 1, Los Reyes Iztacala, Tlalnepantla, Estado de México, México C.P 54090

\begin{abstract}
The brown shrimp (Penaeus aztecus) fishery is the most important in the Gulf of Mexico. This fishery takes non-target species or by-catch fauna, mainly teleost fishes, which are incidentally captured. Some of the by-catch species has commercial use and the rest of them are discarded. Therefore, it is important to describe the composition of the discarded fauna, to determine the impacts under the ecosystem approach. Discarding catch of shrimp trawls carried out in 2013 on the continental shelf off the State of Veracruz in different depth intervals $(22-46$ and $>46 \mathrm{~m}$ ) was analyzed. For each trawl, the shrimp catch, the commercial by-catch and the discarding were recorded and the catch ratios respect to shrimp, were obtained. A discard sample was taken and the species were identified, the total length was taken and the ecological parameters were calculated. The non-parametric multiple dimensional scaling test was performed. A total of 7,178 fishes were collected from 34 families, 46 genera and 51 species. The ratio of commercial species by-catch: shrimp and discard: shrimp was 15.41:1 and 40:1 kg, respectively. The species with high relative dominance were Citharichthys macrops, Synodus foetens, Diplectrum bivittatum, Rhomboplites aurorubens and Upeneus parvus. The species with greater importance index were C. macrops, S. foetens, and D. bivittatum. The $43.14 \%$ of the discarded species are used as food and $7.84 \%$ as bait. The maximum diversity $\left(\mathrm{H}^{\prime}\right)$ was 3.76 bits ind $^{-1}$ and the trophic level was 4.31. The study of discard composition is a basis to assess the impact generated by the shrimp trawlers and provides guidelines for identify changes in the community structure.
\end{abstract}

Key words: Discard, fishes, shrimp, Alvarado, Veracruz

Resumen.- La pesquería del camarón café Penaeus aztecus es la más importante en el Golfo de México. En esta pesquería se capturan de manera incidental especies no objetivo compuestas mayormente por peces teleósteos. Es importante describir la composición del descarte, para determinar el impacto de los arrastres camaroneros bajo un enfoque ecosistémico. Se analizó la captura de descarte de arrastres camaroneros realizados en 2013 en la plataforma continental del Estado de Veracruz en 22-46 y $>46 \mathrm{~m}$ de profundidad. Para cada arrastre se registró la captura de camarón, fauna de acompañamiento comercial, descarte y fauna de acompañamiento. Se tomó una muestra del descarte, se identificaron las especies y se calcularon los parámetros ecológicos de la muestra. Se realizó una prueba de escalamiento dimensional múltiple no paramétrica. De 87 lances de pesca, se analizaron 7.178 organismos correspondientes a 34 familias, 46 géneros y 51 especies. Las especies con mayor dominancia relativa y mayor valor del índice de importancia fueron: Citharichthys macrops, Synodus foetens, Diplectrum bivittatum, Rhomboplites aurorubens y Upeneus parvus. Un alto porcentaje de las especies de descarte $(43,14 \%)$ presentan uso potencial de alimento y 7,84\% carnada. En promedio, la relación Fauna de Acompañamiento Comercial:Camarón y Descarte:Camarón fue de 15,41:1 y $40: 1 \mathrm{~kg}$, respectivamente. El valor máximo de diversidad $\left(\mathrm{H}^{\prime}\right)$ fue de 3,76 bits ind $^{-1}$ y el Nivel Trófico de 4,31. El estudio de la composición de los descartes es base para evaluar el impacto generado por los arrastres de camarón en la costa veracruzana y proporciona pautas para identificar cambios en la estructura de la comunidad.

Palabras clave: Descarte, peces, camarón, Alvarado, Veracruz 


\section{INTRODUCCIÓN}

La pesca de camarón genera importantes beneficios económicos y sociales en países en desarrollo (FAO 2012). En el Golfo de México, esta pesquería ocupa el cuarto lugar por volumen de captura siendo la más importante por valor económico de producción e infraestructura utilizada en su captura y procesamiento (Wakida-Kusunoki et al. 2006, CONAPESCA 2013). Esta pesquería se basa principalmente en el camarón café Penaeus aztecus (Ives, 1891) con 95\% del total del volumen de captura que proviene principalmente de los estados de Tamaulipas y Veracruz, con una flota camaronera aproximada de 200 barcos que operan en los puertos de Tampico, Tuxpan, Veracruz y Alvarado, México (CONAPESCA 2013, SAGARPA 2011).

La pesquería de camarón en Veracruz representó el 10\% de la captura total del Golfo de México (21.700 ton) entre 2004 y 2013 (SAGARPA 2013). Además, genera ingresos económicos y beneficios sociales, e impacta al ecosistema a través de la captura incidental de un número importante de especies no objetivo, en su mayoría, peces teleósteos (Grainger \& García 1996). A este conjunto de especies se les denomina fauna de acompañamiento del camarón (FAC), de la cual una porción es retenida y desembarcada ya sea para uso alimenticio, comercial o como carnada, en tanto que el resto es descartado y devuelto al mar por no presentar tallas comerciales, por no tener algún valor o como resultado de factores económicos, legales y culturales (McCaughran 1992, Alverson et al. 1994).

El enfoque en el estudio de la FAC es diverso, por ejemplo Duarte et al. (2006) la analizan a partir de la estructura de tallas y tasas de capturabilidad y su relación con la actividad circadiana en la pesca de arrastre en Colombia. Por su parte, Báez \& Balsinde (2008), establecen a la talla de captura como la principal razón de las altas tasas de descarte en el Golfo de Nicoya, Costa Rica, con una proporción FAC:camarón de 18,5:1 kg. Así mismo, Valdés et al. (2010, 2011), coinciden en que la talla de captura es la razón principal del descarte y analizan la variabilidad en la composición y estructura de tallas de la FAC. Giménez-Hurtado et al. (2016), analizan la composición de la FAC en Cuba, registrando 41 especies de peces con un intervalo de tamaño de 6 a $34 \mathrm{~cm}$, lo cual plantea un problema para su conservación debido al impacto de esta actividad sobre especies comerciales.

En México, el Instituto Nacional de Pesca y Acuacultura (INAPESCA) ha documentado la relación FAC:camarón desde 1956 a 1996 (Gillett 2008), con aportes acerca de la composición y dinámica de la FAC. Grande-Vidal \& Díaz(1981) reportan una proporción FAC:camarón de 9:1 y 3:1 para el Pacífico y Golfo de México, respectivamente, así como de 10 a 18 especies abundantes sujetas a comercialización.
Los estudios sobre la FAC en México se han centrado en la relación FAC:camarón, sin embargo, se ha documentado la composición y dinámica de la FAC principalmente en el Pacífico. López-Martínez et al. (2010) presentan un listado sistemático de la FAC en el Golfo de California, siendo los peces el grupo dominante, con 158 especies y longitudes desde 2,2 a 94,7 cm. Posteriormente, López-Martínez et al. (2012) para el Golfo de California, resaltan que la mayor problemática es el descarte de juveniles de especies de importancia comercial y que la relación FAC:camarón varía dependiendo de la zona y estación del año.

Los estudios sobre la dinámica de la FAC para el Golfo de México son escasos. Yáñez-Arancibia (1986) reportó una proporción de FAC:camarón para la Sonda de Campeche de 12:1 y 106 especies de peces. Wakida-Kusunoki et al. (2013) analizaron la distribución y abundancia de la FAC en Tamaulipas, registrando 97 especies de peces, la mayoría juveniles y tallas pequeñas, con una relación de 14,8 kg de FAC por $1 \mathrm{~kg}$ de camarón.

Abarca-Arenas et al. (2004) y Peláez-Rodríguez et al. (2005) realizaron análisis tróficos de la comunidad de peces demersales obtenidos de la FAC en Alvarado, Veracruz. Más adelante, Abarca-Arenas et al. (2007), reportaron un análisis sociométrico de la macrofauna asociada a la plataforma continental que es impactada por la actividad de arrastre camaronero.

Tomando en cuenta que la proporción FAC:camarón no es un indicador suficientemente robusto para estimar la magnitud del impacto de la actividad pesquera, debido a la falta de conocimiento sobre la composición de las especies de descarte y que los peces son el principal componente, la remoción de éstos genera un impacto en la comunidad biótica en general y en una amplia gama de interacciones ecológicas en particular (López-Martínez et al. 2012), promoviendo cambios profundos en el ecosistema (Jennings \& Kaiser 1998, Hall 1999).

Debido a lo anterior, los inventarios ictiofaunísticos son un instrumento básico para la administración pesquera a través del enfoque ecosistémico de la pesca (EEP) (RodríguezRomero et al. 2008). Bajo el EEP, el conocimiento de la estructura de la comunidad de peces que compone el descarte y de sus parámetros comunitarios, se ha convertido en una herramienta básica para la evaluación del impacto ambiental de la pesquería (Jennings \& Reynolds 2000). La generación de información biológica y ecológica de este componente aporta elementos de juicio para mejorar el manejo del recurso camarón, considerando la complejidad del escenario en el que se efectúa y los diferentes impactos ambientales que produce el arrastre camaronero (Charles 2001, FAO 2003). 
De acuerdo a lo anterior, se considera que las diferencias en la composición y estructura de la comunidad de peces de descarte están relacionadas con la profundidad y la ubicación geográfica a lo largo de la costa centro-sur de Veracruz, por lo tanto, el presente estudio aporta información sobre la composición y estructura de la comunidad de peces que son parte de la FAC, en específico del descarte, en la zona central del estado de Veracruz.

\section{MATERIALES Y MÉTODOS}

La zona de pesca de camarón para este estudio, se encuentra ubicada en la plataforma continental del suroeste del Golfo de México y se extiende desde el puerto de Alvarado $\left(18^{\circ} 47^{\prime} 42,94^{\prime \prime} \mathrm{N}\right.$ y $\left.95^{\circ} 44^{\prime} 43,87^{\prime \prime O}\right)$ hasta las cercanías de Coatzacoalcos $\left(18^{\circ} 10^{\prime} 50,16^{\prime \prime} \mathrm{N}\right.$ y $\left.94^{\circ} 25^{\prime} 11,51^{\prime \prime O}\right)$, en el estado de Veracruz (Fig. 1). La plataforma continental en esta zona se caracteriza por tener una marcada influencia continental y extensos sistemas estuarino-lagunares. Esta región recibe la influencia del río Papaloapan a través del sistema lagunar de
Alvarado (SLA), caracterizado por ser un sistema deltaico, con un aporte considerable de elementos terrígenos con materia orgánica (Lara-Domínguez et al. 2011). El tipo de sedimento de la zona de pesca es reflejo de la influencia de los sistemas estuarino-lagunares, dominando los sedimentos limo arenosos terrígenos, presentándose una dominancia de fondos planos y en menor proporción, fondos irregulares compuestos de sedimentos muy consolidados y fondos rocosos, materiales de origen marino y costero-aluvial (Jiménez 1979, GonzálezGándara 2011).

El clima de la zona de estudio, de acuerdo con García (1982), es del tipo $\mathrm{Aw}^{2}$ (i'), que corresponde a un clima caliente subhúmedo, con las mayores precipitaciones entre junio y septiembre que varían entre 1,1 y $2,0 \mathrm{~mm}$, con una temperatura media anual de $26,4^{\circ} \mathrm{C}$ y valores máximos y mínimos de 29,3 y $22,6^{\circ} \mathrm{C}$, respectivamente. El comportamiento climático de esta zona permite definir tres temporadas: nortes (octubre a febrero), secas (marzo a mayo) y lluvias (junio a septiembre) (Morán et al. 2005).

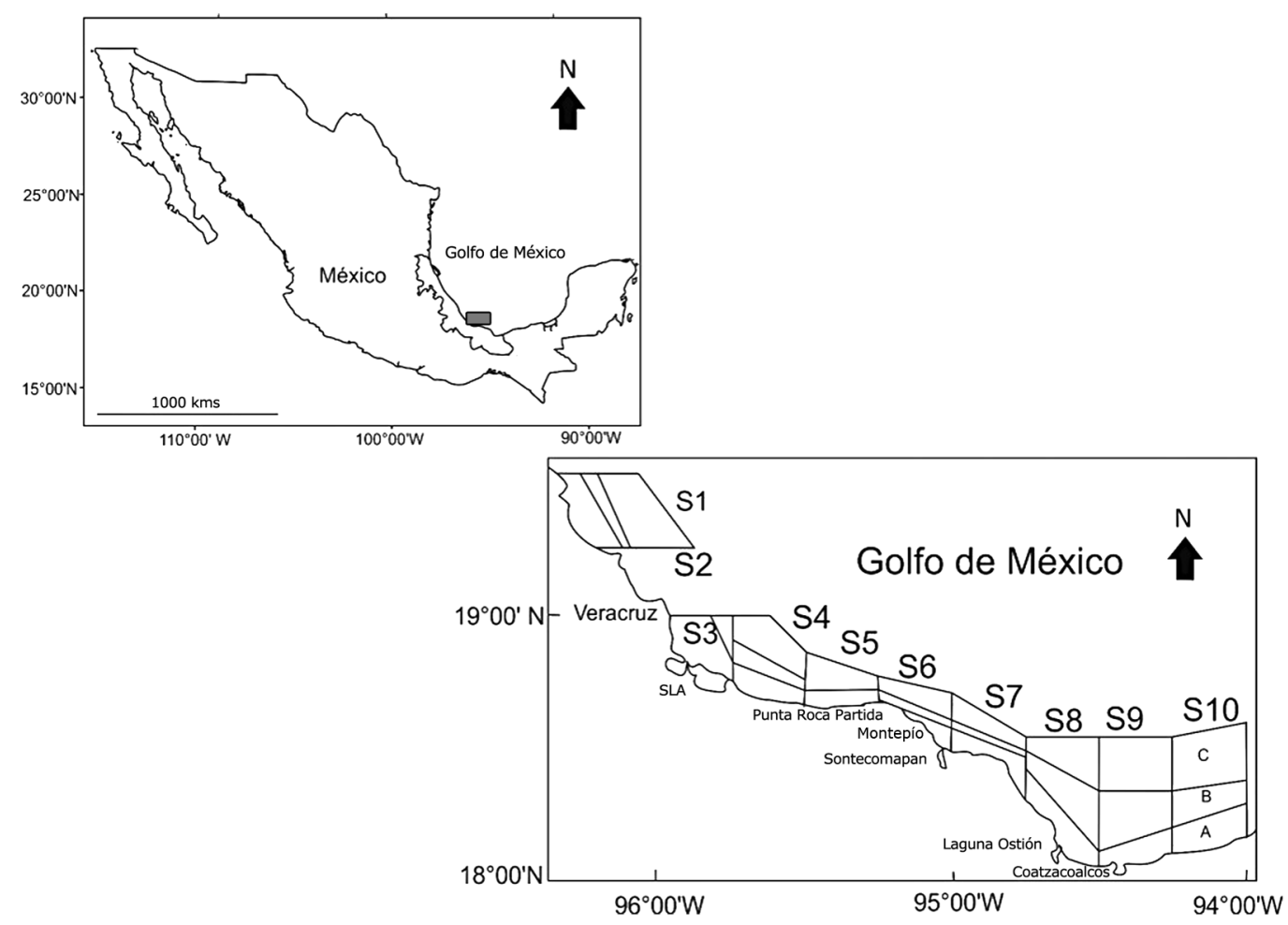

Figura 1. Localización de la zona de estudio. Tomado de SAGARPA-INAPESCA (2010). Dictamen Técnico. Instituto Nacional de Pesca / Study area, taken from SAGARPA-INAPESCA (2010). Dictamen Técnico. Instituto Nacional de Pesca 
Se analizó la información obtenida de 87 arrastres, realizados en junio, julio y octubre de 2013, correspondientes a la temporada de lluvias. Setenta y cinco lances de pesca (26 en junio y 49 en julio) provienen de cruceros de investigación realizados por el Instituto Nacional de Pesca y Acuacultura (Centro Regional de Investigación Acuícola y Pesquera, Veracruz), el cual divide el litoral veracruzano en 25 cuadrantes que siguen el perfil batimétrico con 3 intervalos de profundidad: A) 0 a $22 \mathrm{~m}$, B) 22 a $46 \mathrm{~m}$ y C) > $46 \mathrm{~m}$ (SAGARPAINAPESCA 2010). Los arrastres se ubicaron en 16 cuadrantes, en los bloques de profundidad B y C (Fig. 1). Además, se analizaron 12 arrastres llevados a cabo en octubre en las zonas y temporada regular de pesca comercial, que comprende de agosto a mayo. Ambos tipos de prospecciones fueron realizadas a bordo de embarcaciones camaroneras de la flota comercial que opera en el puerto de Alvarado, Veracruz, que utilizan para su operación 4 redes de arrastre tipo japonesa de pesca múltiple, de $20 \mathrm{~m}$ de largo, $9 \mathrm{~m}$ de abertura de trabajo y luz de malla de $13 / 4$ ". La duración de los arrastres fue de 2 a 6 h, a una velocidad de 3 nudos. Todos los arrastres fueron nocturnos.

Para cada arrastre se registró el peso de la captura total de camarón, FAC (todas las especies capturadas incidentalmente), fauna de acompañamiento comercial 'FACC' (especies que se conservan debido a que tienen algún uso), y descarte (especies de vertebrados que son devueltos al mar), con una báscula colgante modelo CRS200 de capacidad de $200 \mathrm{~kg}$. Las capturas fueron estandarizadas a captura por unidad de tiempo de arrastre tomando como base que los arrastres con menor tiempo fueron de $2 \mathrm{~h}$ (CPUE $\mathrm{kg}^{-2 \mathrm{~h}}$ ), usando el método de área barrida (Sparre \& Venema 1995). A cada conjunto de datos se le aplicó la prueba de normalidad de Shapiro-Wilk (Sokal $\&$ Rohlf 2003), posteriormente se obtuvieron las medidas de tendencia central y de dispersión por cuadrante de pesca.

Se estimaron para cada arrastre la proporción de captura de FACC:Camarón y la proporción Descarte:Camarón. Para la comparación de las proporciones, los datos fueron ponderados considerando el valor más alto en cada sitio, posteriormente fueron transformados con el arcoseno. Para la comparación entre los cuadrantes de pesca se aplicó un análisis de varianza de una vía (ANOVA) (Sokal \& Rohlf 2003).

Para el análisis ecológico del componente íctico, se tomó una muestra del descarte de $4 \mathrm{~kg}$ de cada arrastre efectuado, basado en la disponibilidad de espacio en el cuarto frío de la embarcación, según Giménez-Hurtado et al. (2016), quienes tomaron muestras de $5 \mathrm{~kg}$ por arrastre camaronero en la plataforma suroriental de Cuba. Los organismos recolectados se lavaron y conservaron etiquetados en bolsas de polietileno para su congelación y posterior procesamiento. Se realizaron entrevistas al personal de cubierta (pescadores) durante los viajes de pesca, para conocer el uso potencial de las especies de peces que son parte del descarte (Sampieri et al. 1998).

En el laboratorio, los organismos se determinaron por medio de las claves de identificación de Hoese \& Moore (1998), Castro-Aguirre (1999) y Carpenter (2002). A cada organismo se le registró su longitud total (LT) con ayuda de un ictiómetro de $1 \mathrm{~mm}$ de precisión. Se determinó el nivel trófico(NT) para cada una de las especies de peces del descarte, utilizando la base de datos de Fishbase (Froese \& Pauly 2016). Los nombres comunes se establecieron de acuerdo con Page et al. (2013). La composición de la ictiofauna del descarte se determinó mediante la estimación del índice valor de importancia por especie (IVI), el cual comprendió la suma de los valores relativos de abundancia, frecuencia y dominancia, como la relación de la abundancia relativa de la especie $i$ respecto a la abundancia total de la comunidad, e indica la importancia ecológica relativa de las especies en una comunidad (Matteucci \& Colma 1982). Los indicadores de riqueza de especies de Margalef, diversidad y equitatividad se obtuvieron utilizando el programa PRIMER (v.6) (Hammer \& Harper 2003) de acuerdo con Magurran \& McGill (2011).

Para determinar si la composición de especies cambia a lo largo de los 16 cuadrantes, se realizó la prueba de escalamiento múltiple dimensional no-paramétrica (MDS), considerando los datos de riqueza como número de especies promedio por cuadrante y las similitudes calculadas por el índice de BrayCurtis; así mismo, para apoyar los resultados obtenidos en el MDS se aplicó el análisis de similitud (ANOSIM) para ello se utilizó el programa PRIMER (v.6) (Hammer \& Harper 2003, Chávez-López et al. 2005).

\section{Resultados}

Durante el 2013 se efectuaron un total de 87 arrastres, de los cuales 26 fueron realizados en junio, 49 en julio y 12 en octubre. Veintinueve se ubicaron en el estrato de profundidad B (22-46 m) y 58 en el estrato C (> $46 \mathrm{~m})$.

Los resultados obtenidos en cuanto a las capturas (camarón, FACC, descarte y FAC) presentaron una distribución normal (todos con valores de $P>0,08$ ).

Los valores promedio de CPUE $\mathrm{kg}^{-2 \mathrm{~h}}$ de captura de camarón, fauna de acompañamiento comercial (FACC), descarte y fauna de acompañamiento (FAC) y por estrato de profundidad se presentan en la Figura 2.

La captura máxima de camarón $54,19 \mathrm{~kg}^{-2 \mathrm{~h}}$, se obtuvo en el intervalo de profundidad $\mathrm{B}$, frente a la desembocadura del sistema lagunar de Alvarado (SLA); en tanto que la captura 


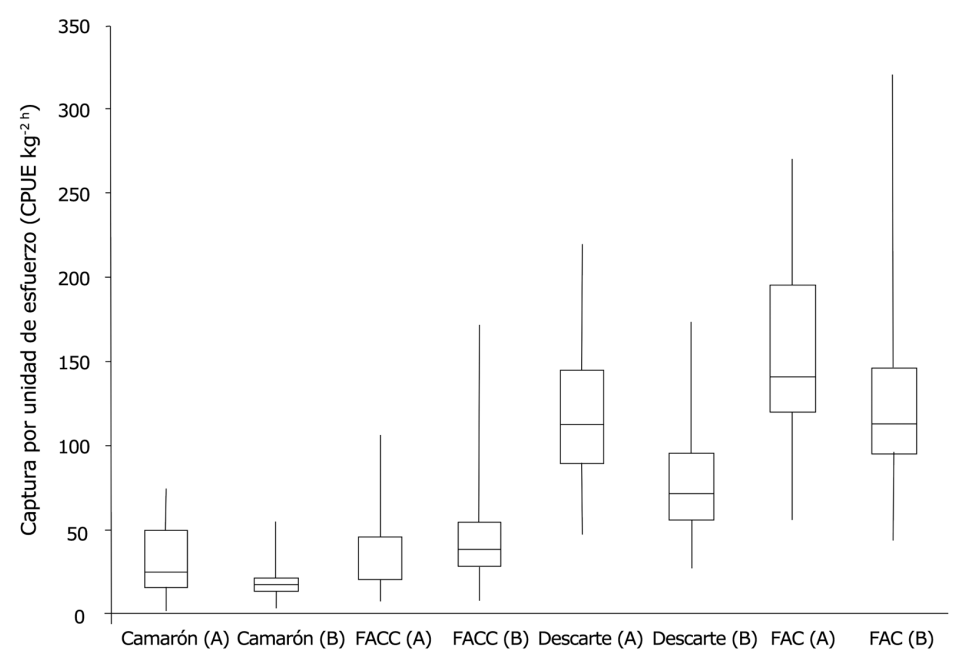

Figura 2. Captura por unidad de esfuerzo (CPUE kg ${ }^{2 \mathrm{~h}}$ ) por intervalo de profundidad de arrastre: A) 22-46 m, B) Mayor a $46 \mathrm{~m}$; para camarón, fauna de acompañamiento de camarón comercial (FACC), descarte y fauna de acompañamiento (FAC). Mediana (línea horizontal dentro de la caja), valores máximos y mínimos (líneas verticales) y cuartiles / Capture per unit of effort (CPUE $\mathrm{kg}^{-2 h}$ ) per trawl depth interval: A) 22-46 m, B) Greater than $46 \mathrm{~m}$; for shrimp, commercial shrimp by-catch (FACC), discard and shrimp by catch (FAC). Median (horizontal line inside the box), maximum and minimum values (vertical lines) and quartiles



Figura 3. Relación fauna de acompañamiento comercial-camarón (FACC:CAM), descarte-camarón (DESC:CAM) y fauna de acompañamiento-camarón (FAC:CAM) (kg) por intervalo de profundidad de arrastre A) 22-46 m y B) Mayor a $46 \mathrm{~m}$. Mediana (línea horizontal dentro de la caja), valores máximos y mínimos (líneas verticales) y cuartiles / Ratio of commercial shrimp by-catch-shrimp (FACC:CAM), discard-shrimp (DESC:CAM) and shrimp by catch-shrimp (FAC:CAM) (kg) per depth trawl interval A) 22-46 m and B) Greater than $46 \mathrm{~m}$. Median (horizontal line inside the box), maximum and minimum values (vertical lines) and quartiles

mínima se ubicó al norte del puerto de Veracruz con $3,00 \mathrm{~kg}^{-2 \mathrm{~h}}$, esta última caracterizada por no ser una zona habitual de pesca de camarón. El valor promedio de camarón para los estratos de profundidad B y C fue de $24,60 \pm 19,93 \mathrm{~kg}^{-2 \mathrm{~h}}$ y $18,89 \pm$ $8,30 \mathrm{~kg}^{-2 \mathrm{~h}}$, respectivamente, sin embargo, se presentaron diferencias estadísticamente significativas entre los estratos de profundidad $(P<0,001)$. El descarte presentó una captura máxima de $160,00 \mathrm{~kg}^{-2 \mathrm{~h}}$, con valores promedio de 123,53 \pm $24,61 \mathrm{~kg}^{-2 \mathrm{~h}}$ y $89,18 \pm 27,49 \mathrm{~kg}^{-2 \mathrm{~h}}$ para los intervalos de profundidad $\mathrm{B}$ y C, respectivamente. En la comparación entre los intervalos de profundidad se presentaron diferencias estadísticamente significativas $(P<0,001)$. La FAC registró valores promedio para los intervalos de profundidad $\mathrm{B}$ y $\mathrm{C}$ de $170,87 \pm 49,90 \mathrm{~kg}^{-2 \mathrm{~h}}$ y $154,75 \pm 55,26 \mathrm{~kg}^{-2 \mathrm{~h}}$, respectivamente 
(Fig. 2). Es importante mencionar que se obtuvo la captura máxima deFAC, $263,30 \mathrm{~kg}^{-2 h}$, en una zona donde habitualmente no se pesca camarón, al norte del puerto de Veracruz (cuadrante $\mathrm{S} 1)$; en esta comparación se encontraron diferencias estadísticamente significativas $(P=0,009)$. La CPUE para la FACC presentó promedios de 47,34 $\pm 28,95 \mathrm{~kg}^{-2 \mathrm{~h}}$ en el intervalo de profundidad B y $65,57 \pm 31,40 \mathrm{~kg}^{-2 \mathrm{~h}}$ para el intervalo C, con un registro máximo de $123,30 \mathrm{~kg}^{-2 \mathrm{~h}}$, ubicado al norte del puerto de Veracruz, así mismo no se presentaron diferencias estadísticamente significativas para los intervalos de profundidad $(P=0,232)$.

La relación FACC:Camarón presentó valores entre 8,20:1 a 0,60:1 con valor promedio de 4,09:1 para el intervalo de profundidad $\mathrm{B}$, en tanto que para el intervalo de profundidad C varío entre 15,41:1 y 1,89:1, con un promedio de 4,60:1 (Fig. 3). La relación Descarte:Camarón presentó un valor máximo de 40,00:1 kg correspondiente al intervalo de profundidad B y un mínimo de 2,48:1, frente al SLA y 12,36:1 como valor promedio. El intervalo de profundidad $\mathrm{C}$ presentó valores entre 17,50:1 a 3,05:1, con un valor promedio de 6,49:1 (Fig. 3). En el intervalo de profundidad B se obtuvo la relación más alta deFAC:Camarón con 46,83:1 kg y 3,08:1 como valor mínimo. En tanto que el intervalo de profundidad C presentó un valor promedio de 11,09:1 con un máximo de 32,91:1 y un mínimo de 5,33:1 (Fig. 3). El análisis estadístico realizado entre las proporciones, mostró que en todas las comparaciones que se hicieron entre los dos intervalos de profundidad, no presentaron diferencias estadísticamente significativas (FACC:Camarón, $P=0,759$; Descarte:Camarón, $P=0,116$; FAC:Camarón, $P=0,285)$.

Se analizaron 7.178 organismos que corresponden a 34 familias, 46 géneros y 51 especies en 87 arrastres realizados. La dominancia del descarte incide en 5 familias que suman el $69,76 \%$ de la abundancia total, correspondiendo a las familias Paralichthyidae (23,53\%), Synodontidae (22,23\%), Serranidae $(11,78 \%)$, Gerridae $(6,44 \%)$ y Lutjanidae $(5,78 \%)$, en tanto que las 29 familias restantes suman el 30,24\% (Tabla 1). a)

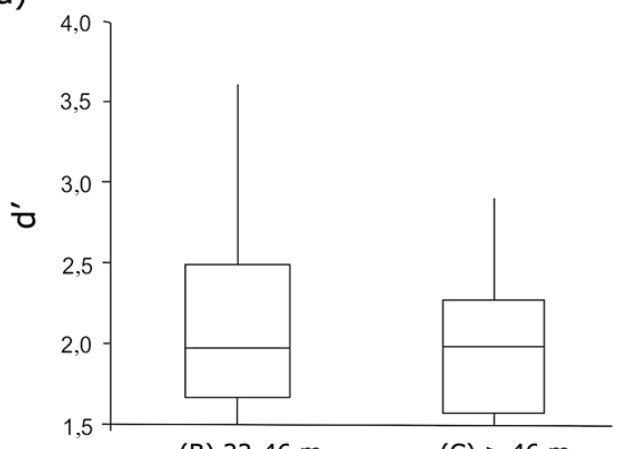

c)

(B) $22-46 \mathrm{~m}$

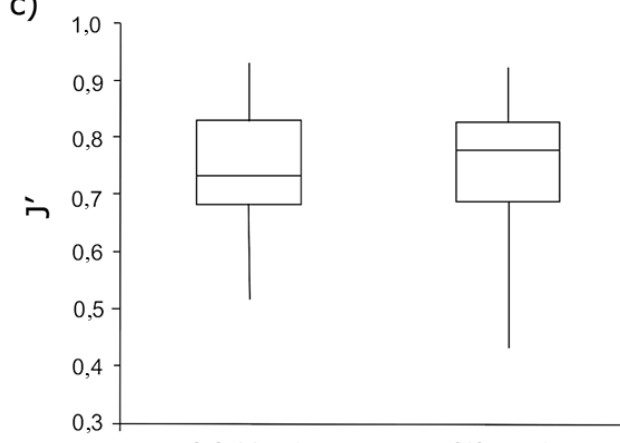

b)

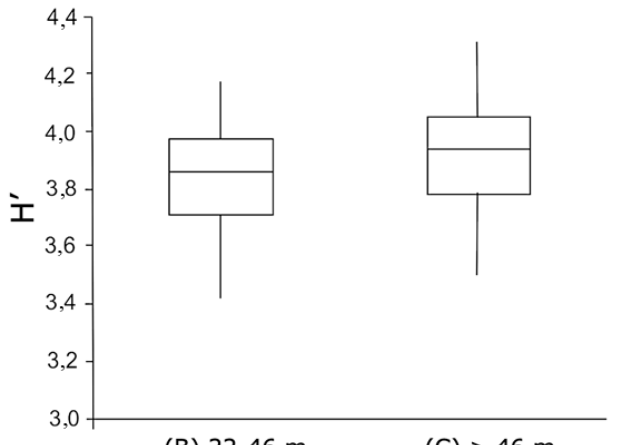

d)

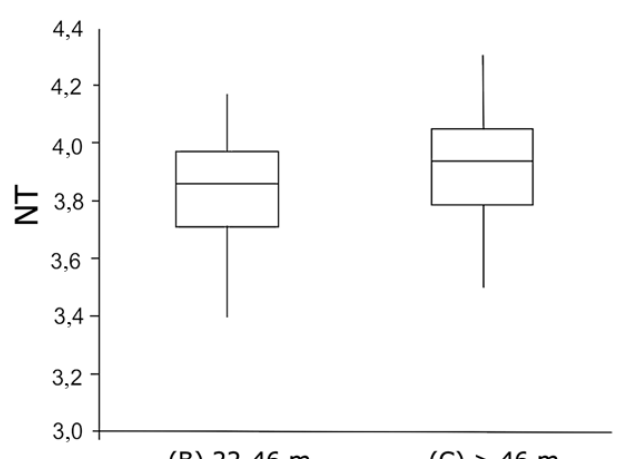

Figura 4. Valores obtenidos por intervalo de profundidad de arrastre (B, C): a) Riqueza de especies (d'), b) Diversidad $\left(H^{\prime}\right)$ bits ind $\left.^{-1}, C^{\prime}\right)$ Equitatividad ( ${ }^{\prime}$ ) y d) Nivel Trófico (NT). Mediana (línea horizontal dentro de la caja), valores máximos y mínimos (líneas verticales) y cuartiles / Values obtained by trawl depth interval $(B, C)$ : a) Species richness ( $\left.d^{\prime}\right)$, b) Diversity $\left(H^{\prime}\right)$ bits ind $\left.{ }^{-1}, c\right)$ Evenness $\left(J^{\prime}\right)$ and d) Trophic Level (NT). Median (horizontal line inside the box), maximum and minimum values (vertical lines) and quartiles 
Tabla 1. Elenco sistemático de la fauna de descarte de arrastres camaroneros en la zona centro-sur del estado de Veracruz. Nombre común, frecuencia relativa (Frec. Rel.), dominancia relativa (Dom. Rel.), índice de valor de importancia (IVI), intervalo de longitud total (LT), nivel trófico (NT), uso de las especies ( $B=$ carnada, $C=$ comercial y $D=$ descarte) y tipo de hábitat / Systematic survey of shrimp trawl discard fauna in the central-southern zone of the state of Veracruz. Common name, relative frequency (Frec. Rel.) relative dominance (Dom. Rel.), Importance Value Index (IVI), trophic level (NT), total length range (LT), species use (B= bait, $C=$ commercial and $D=$ discard) and type of habitat collected by species

\begin{tabular}{|c|c|c|c|c|c|c|c|c|}
\hline Especie & Nombre común & $\begin{array}{c}\text { Frec. } \\
\text { Rel. }\end{array}$ & $\begin{array}{l}\text { Dom. } \\
\text { Rel. }\end{array}$ & IVI & $\begin{array}{l}\mathrm{LT} \\
(\mathrm{cm})\end{array}$ & NT & Uso & Hábitat \\
\hline \multicolumn{9}{|l|}{ RAJIDAE } \\
\hline Raja texana & raya tigre & 2,01 & 0,34 & 2,35 & $7,0-17,0$ & 3,8 & $\mathrm{D}$ & Demersal \\
\hline \multicolumn{9}{|l|}{ MURAENIDAE } \\
\hline Gymnothorax nigromarginatus $\mathrm{r}$ & morena de margen negro & 0,24 & 0,03 & 0,26 & $30,5-40,0$ & 4,1 & $\mathrm{D}$ & Demersal \\
\hline \multicolumn{9}{|c|}{ OPHICHTHIDAE } \\
\hline Myrophis punctatus & tieso gusano & 0,12 & 0,01 & 0,13 & 23 & 4,1 & $\mathrm{D}$ & Demersal \\
\hline \multicolumn{9}{|l|}{ ENGRAULIDAE } \\
\hline Anchoa hepsetus & anchoa legítima & 0,36 & 0,07 & 0,42 & $10,0-10,5$ & 3,3 & $\mathrm{C}$ & Pelágico \\
\hline \multicolumn{9}{|l|}{ CLUPEIDAE } \\
\hline Harengula jaguana & sardinita vivita escamuda & 1,18 & 0,45 & 1,63 & $10,5-14,5$ & 3,3 & B & Demersal \\
\hline Sardinella aurita & sardina española & 0,59 & 0,11 & 0,70 & $13,0-16,0$ & 3,4 & B & Pelágico \\
\hline \multicolumn{9}{|l|}{ SYNODONTIDAE } \\
\hline Synodus foetens & chile apestoso & 9,24 & 22,23 & 31,47 & $4,0-68,0$ & 4,5 & B & Demersal \\
\hline \multicolumn{9}{|l|}{ OPHIDIIDAE } \\
\hline Lepophidium brevibarbe & congriperla clarín & 1,07 & 0,19 & 1,26 & $8,0-25,0$ & 3,7 & $\mathrm{D}$ & Demersal \\
\hline Lepophidium graellsi & congriperla & 1,66 & 0,43 & 2,09 & $11,0-24,0$ & 3,6 & $\mathrm{D}$ & Demersal \\
\hline \multicolumn{9}{|l|}{ BATRACHOIDIDAE } \\
\hline Porichthys porosissimus & sapo & 2,61 & 0,61 & 3,22 & $9,0-15,5$ & 3,7 & $\mathrm{D}$ & Demersal \\
\hline \multicolumn{9}{|l|}{ ANTENNARIIDAE } \\
\hline Antennarius striatus & ranisapo estriado & 0,47 & 0,28 & 0,76 & $2,5-5,5$ & 4,2 & $\mathrm{D}$ & Bentónico \\
\hline \multicolumn{9}{|l|}{ OGCOCEPHALIDAE } \\
\hline Halieutichthys aculeatus & murciélago picudo & 0,36 & 0,08 & 0,44 & $2,0-4,0$ & 3,3 & $\mathrm{D}$ & Bentónico \\
\hline \multicolumn{9}{|l|}{ SCORPAENIDAE } \\
\hline Scorpaena calcarata & escorpión pelón & 7,94 & 4,60 & 12,54 & $5,0-9,0$ & 3,5 & $\mathrm{D}$ & Demersal \\
\hline Scorpaena plumieri & escorpión negro & 0,71 & 0,84 & 1,55 & $19,0-30,0$ & 3,8 & $\mathrm{C} / \mathrm{D}$ & Demersal \\
\hline \multicolumn{9}{|l|}{ TRIGLIDAE } \\
\hline Bellator militaris & rubio soldadito & 1,66 & 0,93 & 2,59 & $3,0-8,5$ & 3,4 & $\mathrm{D}$ & Demersal \\
\hline Prionotus punctatus & rubio azul & 3,91 & 0,97 & 4,88 & $7,0-39,0$ & 3,8 & $\mathrm{C} / \mathrm{D}$ & Demersal \\
\hline Prionotus stearnsi & rubio pequeño & 3,08 & 1,29 & 4,37 & $18,0-38,0$ & 3,6 & $\mathrm{D}$ & Demersal \\
\hline \multicolumn{9}{|l|}{ PERISTEDIIDAE } \\
\hline Peristedion gracile & vaquita blindada flaca & 0,59 & 0,26 & 0,85 & $5,0-7,0$ & 3,4 & $\mathrm{D}$ & Batidemersal \\
\hline \multicolumn{9}{|l|}{ SERRANIDAE } \\
\hline Diplectrum bivittatum & serrano guavino & 7,11 & 11,78 & 18,89 & $3,5-12,0$ & 4,0 & $\mathrm{C} / \mathrm{D}$ & Demersal \\
\hline \multicolumn{9}{|l|}{ PRIACANTHIDAE } \\
\hline Priacanthus arenatus & catalufa ojona & 0,24 & 0,03 & 0,26 & $15,0-28,0$ & 4,0 & $\mathrm{C} / \mathrm{D}$ & Demersal \\
\hline \multicolumn{9}{|l|}{ MALACANTHIDAE } \\
\hline Caulolatilus cyanops & domingo & 0,24 & 0,03 & 0,26 & $10,5-21,0$ & 4,0 & $\mathrm{C}$ & Demersal \\
\hline \multicolumn{9}{|l|}{ CARANGIDAE } \\
\hline Decapterus punctatus & macarela chuparaco & 0,12 & 0,04 & 0,16 & $12,0-14,5$ & 4,3 & $\mathrm{C}$ & Pelágico \\
\hline Selar crumenophthalmus & charrito ojón & 3,79 & 2,57 & 6,36 & $9,5-14,5$ & 4,1 & $\mathrm{D}$ & Demersal \\
\hline Selene vomer & jorobado penacho & 1,18 & 1,37 & 2,55 & $12,0-16,0$ & 4,3 & $\mathrm{D}$ & Demersal \\
\hline Trachurus lathami & charrito garretón & 2,13 & 1,31 & 3,45 & $6,5-18,0$ & 4,0 & $\mathrm{D}$ & Bentopelágico \\
\hline \multicolumn{9}{|l|}{ LUTJANIDAE } \\
\hline Lutjanus campechanus & huachinango del Golfo & 0,95 & 0,18 & 1,12 & $18,0-19,0$ & 4,0 & $\mathrm{C} / \mathrm{D}$ & Demersal \\
\hline Lutjanus synagris & pargo biajaiba & 0,36 & 0,04 & 0,40 & $14,0-29,0$ & 3,8 & $\mathrm{C} / \mathrm{D}$ & Demersal \\
\hline Rhomboplites aurorubens & besugo & 8,53 & 5,56 & 14,09 & $4,5-20,5$ & 4,3 & $\mathrm{C} / \mathrm{D}$ & Demersal \\
\hline
\end{tabular}




\section{Tabla 1. Continuación / Continued}

\begin{tabular}{|c|c|c|c|c|c|c|c|c|}
\hline Especie & Nombre común & $\begin{array}{c}\text { Frec. } \\
\text { Rel. }\end{array}$ & $\begin{array}{c}\text { Dom. } \\
\text { Rel. }\end{array}$ & IVI & $\begin{array}{l}\mathrm{LT} \\
(\mathrm{cm})\end{array}$ & NT & Uso & Hábitat \\
\hline \multicolumn{9}{|l|}{ GERREIDAE } \\
\hline Diapterus auratus & mojarra guacha & 0,95 & 0,70 & 1,65 & $9,0-11,5$ & 2,4 & $\mathrm{C}$ & Demersal \\
\hline Eucinostomus melanopterus & mojarra de ley & 3,44 & 5,25 & 8,69 & $9,0-13,5$ & 3,4 & $\mathrm{C}$ & Demersal \\
\hline Eugerres plumieri & mojarra rayada & 0,95 & 0,49 & 1,44 & $9,0-12,5$ & 2,2 & $\mathrm{C}$ & Demersal \\
\hline \multicolumn{9}{|l|}{ HAEMULIDAE } \\
\hline Haemulon aurolineatum & ronco jeníguaro & 3,32 & 3,57 & 6,89 & $3,5-16,0$ & 3,2 & $\mathrm{C} / \mathrm{D}$ & Demersal \\
\hline \multicolumn{9}{|l|}{ SPARIDAE } \\
\hline Stenotomus caprinus & sargo espinudo & 1,42 & 0,39 & 1,81 & $1,5-16,0$ & 3,1 & $\mathrm{D}$ & Demersal \\
\hline \multicolumn{9}{|l|}{ POLYNEMIDAE } \\
\hline Polydactylus octonemus & barbudo ocho barbas & 0,47 & 0,15 & 0,62 & $12,0-13,5$ & 3,6 & $\mathrm{D}$ & Demersal \\
\hline \multicolumn{9}{|l|}{ SCIAENIDAE } \\
\hline Cynoscion nothus & corvina plateada & 0,83 & 0,20 & 1,03 & $17,0-42,5$ & 4,0 & $\mathrm{C}$ & Demersal \\
\hline Larimus fasciatus & boquinete listado & 0,83 & 0,23 & 1,06 & $14,0-29,0$ & 3,6 & $\mathrm{D}$ & Demersal \\
\hline Stellifer lanceolatus & corvinilla lanza & 0,24 & 0,35 & 0,59 & $10,0-11,0$ & 3,5 & $\mathrm{D}$ & Demersal \\
\hline Menticirrhus saxatilis & berrugato ratón & 0,12 & 0,03 & 0,15 & $9,5-21,5$ & 3,6 & $\mathrm{C} / \mathrm{D}$ & Demersal \\
\hline Menticirrhus americanus & berrugato zorro & 0,71 & 0,19 & 0,90 & $16,5-37,0$ & 3,5 & $\mathrm{C} / \mathrm{D}$ & Demersal \\
\hline \multicolumn{9}{|l|}{ MULLIDAE } \\
\hline Upeneus parvus & chivo rayuelo & 7,94 & 5,41 & 13,35 & $5,5-15,0$ & 3,9 & B & Demersal \\
\hline \multicolumn{9}{|l|}{ SPHYRAENIDAE } \\
\hline Sphyraena guachancho & tolete & 0,24 & 0,04 & 0,28 & $22,0-26,5$ & 3,9 & $\mathrm{C} / \mathrm{D}$ & Pelágico \\
\hline \multicolumn{9}{|l|}{ TRICHIURIDAE } \\
\hline Trichiurus lepturus & sable del Atlántico & 0,59 & 0,09 & 0,69 & $44,0-45,0$ & 4,5 & $\mathrm{C} / \mathrm{D}$ & Bentopelágico \\
\hline \multicolumn{9}{|l|}{ SCOMBRIDAE } \\
\hline Scomberomorus maculatus & sierra común & 0,24 & 0,16 & 0,40 & $16,5-20,5$ & 4,5 & $\mathrm{C} / \mathrm{D}$ & Pelágico \\
\hline \multicolumn{9}{|l|}{ PARALICHTHYIDAE } \\
\hline Citharichthys macrops & lenguado manchado & 10,07 & 23,53 & 33,60 & $4,0-34,0$ & 3,5 & $\mathrm{C}$ & Bentónico \\
\hline \multicolumn{9}{|l|}{ ACHIRIDAE } \\
\hline Achirus lineatus & suela listada & 1,42 & 0,37 & 1,79 & $7,5-15,0$ & 3,7 & $\mathrm{D}$ & Demersal \\
\hline \multicolumn{9}{|l|}{ CYNOGLOSSIDAE } \\
\hline Symphurus plagiusa & lengua gris & 1,54 & 0,30 & 1,84 & $13,0-15,0$ & 3,0 & $\mathrm{D}$ & Demersal \\
\hline \multicolumn{9}{|l|}{ BALISTIDAE } \\
\hline Balistes vetula & cochino & 0,47 & 0,05 & 0,53 & $14,0-55,0$ & 3,4 & $\mathrm{C}$ & Bentónico \\
\hline \multicolumn{9}{|l|}{ MONOCANTHIDAE } \\
\hline Aluterus schoepfii & lija naranja & 0,12 & 0,01 & 0,13 & $4,0-15,0$ & 2,8 & $\mathrm{D}$ & Demersal \\
\hline \multicolumn{9}{|l|}{ OSTRACIIDAE } \\
\hline Lactophrys trigonus & chapín búfalo & 0,12 & 0,04 & 0,16 & $19,0-22,0$ & 3,3 & $\mathrm{D}$ & Demersal \\
\hline \multicolumn{9}{|l|}{ TETRAODONTIDAE } \\
\hline Lagocephalus laevigatus & botete grande & 0,24 & 0,66 & 0,90 & $4,5-9,5$ & 4,0 & $\mathrm{D}$ & Pelágico \\
\hline Sphaeroides nephelus & botete fruta & 1,30 & 1,14 & 2,44 & $2,0-11,0$ & 2,4 & $\mathrm{D}$ & Bentónico \\
\hline
\end{tabular}

Las especies del descarte que registraron mayor abundancia relativa fueron Citharichthys macrops (23,53\%), Synodus foetens $(22,23 \%$ ) y Diplectrum bivittatum $(11,78 \%)$ (Tabla 1). En lo referente al índice de valor de importancia (IVI), las 6 especies con los mayores valores fueron Citharichthys macrops (33,60\%), Synodus foetens (31,47\%), Diplectrum bivittatum (18,89\%), Rhomboplites aurorubens (14,09\%),
Upeneus parvus $(13,35 \%)$ y Scorpaena calcarata $(12,54 \%)$ sumando $123,54 \%$ del total del IVI. Veintitrés especies representaron el $66,07 \%$ del IVI con un intervalo de valores entre $8,69 \%$ y $1,03 \%$. Las 22 especies restantes presentaron valores menores a $1 \%$ y representaron el $12,08 \%$ del IVI (Tabla 1). 
La longitud total promedio de los peces de descarte fue de $12,74 \pm 6,35 \mathrm{~cm}$, en tanto que el valor máximo fue de 68,00 $\mathrm{cm}$ y el mínimo de $1,50 \mathrm{~cm}$. Por lo que respecta a las tallas de las especies con mayor valor de importancia (IVI) los promedios se presentaron de la siguiente manera: Citharichthys macrops $12,96 \pm 6,42 \mathrm{~cm}$, Synodus foetens $14,05 \pm 5,70 \mathrm{~cm}$, Diplectrum bivittatum 7,84 $\pm 2,10 \mathrm{~cm}$, Rhomboplites aurorubens 10,84 $\pm 1,66 \mathrm{~cm}$, Upeneus parvus 11,36 $\pm 1,55$ cm y Scorpaena calcarata con $8,69 \pm 2,39 \mathrm{~cm}$.

Conforme a la información obtenida de las entrevistas realizadas a los miembros de la tripulación de los buques camaroneros, de las 51 especies registradas en este estudio, se pudo determinar el uso potencial de 26 especies (consumo humano o carnada) una vez que alcanzan talla comercial (Tabla 1).

La variación del nivel trófico promedio del descarte (Fig. 4d), presentó valores máximos de 4,17 a 4,31 para los intervalos de profundidad de arrastre de 22-46 $\mathrm{m}$ y $>46$ $\mathrm{m}$, respectivamente. Ademas, se encontraron diferencias estadísticamente significativas $(P=0,021)$.

La riqueza expresada como número de especies, osciló entre 6 y 17, en el intervalo de profundidad B se registró el valor máximo; en tanto que, el valor promedio para el mismo intervalo fue de $10,02 \pm 2,04$ y de $9,81 \pm 0,85$ para el intervalo $C$. Sin embargo, no se encontraron diferencias estadísticamente significativas $(P=0,102)$.

En tanto que los valores del índice de riqueza de Margalef oscilaron de 3,61 a 1,10 para los intervalos de profundidad B y $C$, con un valor promedio de 2,03 $\pm 0,44$ y 2,03 $\pm 0,20$, respectivamente para ambos intervalos (Fig. 4a), no existiendo diferencias significativas $(P=0,182)$. Los valores de diversidad $\left(\mathrm{H}^{\prime}\right)$ oscilaron entre 1,78 y 3,76 bits ind $^{-1}$ correspondientes al intervalo de profundidad de 22 a $46 \mathrm{~m}$ (B) y $>46 \mathrm{~m}$ (C) y con valores promedio de 2,42 $\pm 0,34$ bits ind $^{-1}$ y 2,49 $\pm 0,21$ bits ind $^{-1}$, respectivamente (Fig. 4b), no presentando diferencias estadísticamente significativas $(P=0,591)$. En lo referente a la equitatividad, el valor máximo se obtuvo en el intervalo de profundidad B con 0,93 y el mínimo en el intervalo $\mathrm{C}$ con 0,43 (Fig. 4c), sin que se presentaran diferencias estadísticamente significativas $(P=0,585)$.

El diagrama obtenido por la prueba de escalamiento múltiple dimensional no-paramétrico (MDS) (Fig. 5) presentó 3 agrupamientos principales: el primero corresponde a los cuadrantes S7C, S8B, S9B, S6C, S4C, S5C y S9C, y el segundo incluyó a los cuadrantes que se encuentran con una mayor similitud, entre ellos (S4B y S8C) y un bloque de 4 cuadrantes (S6B, S1B y S5B, S7B). Los cuadrantes S10B y
S3B se separaron de los grupos principales. El cuadrante S1C, fue el menos similar en comparación con los demás cuadrantes. El análisis de similitud (ANOSIM) apoyó los resultados obtenidos con el MDS (R=0,804, $P=0,001)$.

\section{Discusión}

De manera general no se encontraron diferencias significativas entre los datos de captura de camarón y FAC considerando los intervalos de profundidad, esto también fue encontrado por Wakida-Kusunoki et al. (2013), sin embargo en el presente estudio hubo diferencias con respecto a los 2 estratos de profundidad entre los valores de captura de camarón, descarte, FAC y nivel trófico. Las diferencias se relacionan con la influencia continental que adiciona especies estuarinodependientes a la comunidad demersal marina (YáñezArancibia 1986), como consecuencia de la movilidad de las especies y del flujo de materia entre sistemas costeros (AbarcaArenas et al. 2012).

La captura por unidad de esfuerzo estandarizada y segregada para los diferentes componentes (camarón, descarte y FAC) estimados en la zona de estudio, presentaron diferencias significativas a lo largo de la zona de pesca y estuvo dominada, en todos los casos por la captura de FAC, seguida por la de descarte, FACC y por último el camarón con los menores rendimientos. La captura de descarte, se caracterizó principalmente por una distribución de tamaños sesgada hacia tallas pequeñas, lo que concuerda con lo expuesto por Clucas (1998) y Wells et al. (2008), quienes refieren que en el Golfo de México el hábitat de la ictiofauna de la captura incidental coincide sobre las áreas de arrastre camaronero, compartiendo espectros de talla e historia de vida con el camarón, haciendo inevitable su captura.

La variación de valores obtenidos en cuanto a la proporción camarón con la FACC, descarte y FAC, en el presente estudio, se corresponde con lo reportado en el Golfo de California por López-Martínez et al. (2012), quienes encontraron alta variabilidad en sus registros, con valores extremos que van de 1,8 a $107 \mathrm{~kg}$ de FAC por kilo de camarón. Sin embargo, el valor medio de Descarte:Camarón difiere notoriamente de lo obtenido previamente por Alverson et al. (1994) para el Golfo de México, quienes estimaron una proporción de Descarte:Camaron de 10,3:1 kg, siendo tan solo el $25 \%$ de lo encontrado en el presente estudio. Los registros aquí descritos, también se encuentran por encima de lo reportado por Slavin (1981) para mares tropicales (10:1), por Madrid-Vera et al. (2007) para el Pacífico mexicano (6 a 31:1), Grande-Vidal \& Díaz (1981) en el Golfo de México (3:1) y Wakida-Kusunoki et al. (2013) con 14,8:1 en Tamaulipas. 


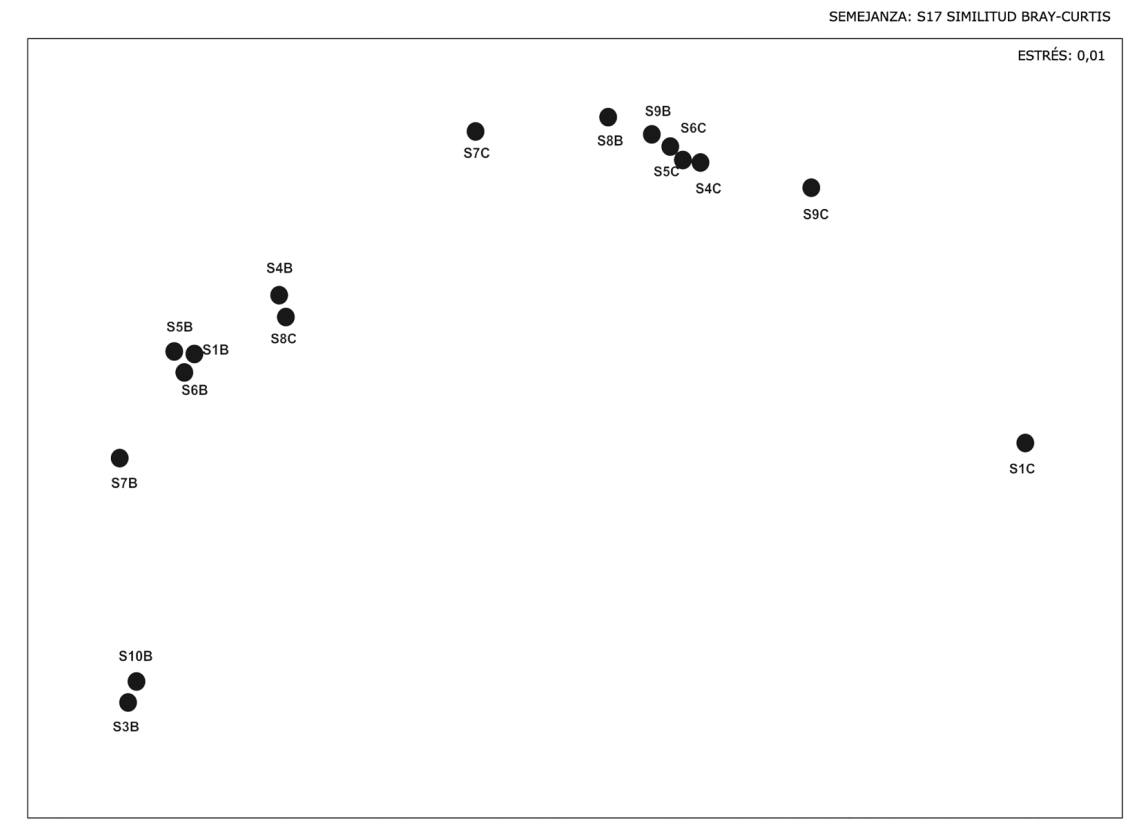

Figura 5. Diagrama de escalamiento múltiple dimensional no-paramétrico (MDS) correspondiente a los 16 cuadrantes de arrastre camaronero. La distancia en el diagrama está representada por los valores de similitud relativa de Bray-Curtis. Los cuadrantes que se encuentran más cercanos unos de otros son más similares. Representación de la relación entre los cuadrantes de arrastre con un alto nivel de exactitud (estrés= 0,01) / Non-parametric multi-dimensional scaling diagram (MDS) corresponding to the 16 quadrants of shrimp trawling. The distances in the diagram are represented by the values of relative similarity of Bray-Curtis. The quadrants that are closest to each other are more similar. Representation of the relationship between the trailing quadrants with a high level of accuracy (stress=0.01)

La proporción de capturas por sí misma no puede ser el principal indicador para la evaluación de los cambios en la estructura de la comunidad de peces como consecuencia del arrastre camaronero, ya que como lo mencionan LópezMartínez et al. (2012b) aún existe una falta de comprensión de la FAC, así como de su variación temporal. En este estudio, para las costas de Veracruz, la relación FAC:Camarón presentó también valores altos y variabilidad importante, ya que el recurso camarón se pesca en zonas costeras, donde ocurre alta productividad primaria (Lohrenz et al. 1999) y por tanto, donde es más probable que se encuentren altas concentraciones de peces (Yáñez-Arancibia 1986). En función de lo anterior será necesario, como lo mencionan Herrera-Valdivia et al. (2015), desarrollar estudios continuos para identificar las especies críticas que son afectadas en su abundancia relativa por efecto del arrastre camaronero y que pueden perturbar a la estructura de la comunidad, en combinación con métodos de análisis multifactoriales basados en índices ecológicos y taxonómicos como una aproximación al conocimiento del estado de salud del ecosistema marino en el largo plazo.
A pesar de que la composición del descarte es específica para cada pesquería y zona de pesca, en riqueza de especies y estructura, la pesca de arrastre camaronero es la que genera un mayor impacto a las comunidades marinas (Vianna \& Almeida 2005). Lo anterior es relevante si se toma en cuenta que los peces son el principal componente de las capturas incidentales en el Golfo de México con cerca del $60 \%$ del total (Scott-Denton et al. 2012). La composición máxima reportada hasta el momento para la pesca incidental para el Golfo de México es de 185 especies (Scott-Denton et al. 2012), de las cuales entre 97 (Wakida-Kusunoki et al. (2013) y 151 (Abarca-Arenas et al. 2004) son peces. De las 51 especies registradas en este estudio, 26 especies son de uso potencial (consumo humano o carnada) una vez que alcanzan talla comercial. Resalta el hecho que varias de éstas especies son consideradas como parte de la pesca ribereña y son objeto de algún tipo de monitoreo en el Golfo de México, consignado a través de la Carta Nacional Pesquera (DOF 2012) ${ }^{1}$.

${ }^{1}$ DOF. 2012. Actualización de la Carta Nacional Pesquera, 24 pp. Diario Oficial de la Federación, México. 
La información sobre la composición de los descartes y tallas generalmente es escasa, por lo que recientemente se ha empezado a incorporar y discutir este tipo de información, así como sus implicaciones para las poblaciones sujetas a explotación pesquera (Bouchon-Navaro et al. 2006). La composición del descarte en la temporadas de lluvias, estuvo dominada por especies demersales, comunes para esta región del Golfo de México (Monk et al. 2015). Citharichthys macrops, Synodus foetens, y Diplectrum bivittatum, constituyen las especies más importantes de este rubro y en consecuencia, son determinantes en la composición y estructura del componente íctico del descarte, característico de arrastres camaroneros en zonas tropicales (Clucas 1998). Duarte et al. (2010) reportaron que las capturas anuales de la FAC en el Caribe Colombiano, están dominadas por 10 grupos, entre ellos Synodus spp., lenguados Syacium spp., mojarras Eucinostomus spp. y pargos Lutjanus synagris, lo cual coincide parcialmente con lo obtenido en este estudio, así como la dominancia de tallas pequeñas en el descarte, lo cual sugiere, que al igual que en el Caribe colombiano, el arrastre camaronero afecta la estructura de las comunidades de peces, manifestándose a través de las tallas de captura (Duarte et al. 2010).

La tendencia a concentrar en pocas familias una alta proporción de la abundancia relativa de la FAC del arrastre camaronero en general, es consecuencia de que la dominancia se concentre en pocas especies en cada localidad. De ello han dado cuenta Rábago-Quiroz et al. (2011) en el Golfo de California, Casteló-Báez \& Balsinde (2008) en el Golfo de Nicoya, Costa Rica, Valdés et al. (2011) en el Golfo de Ana María, Cuba y Peláez-Rodríguez et al. (2005) en Alvarado, Veracruz, dónde la composición de la FAC estuvo dominada por no más de 5 especies.

De acuerdo con Páramo et al. (2009), las pesquerías demersales se caracterizan por ser multiespecíficas y ejercen una presión pesquera considerable sobre un elenco amplio de especies y tallas, bajo riesgo de sobreexplotación de algunas de ellas, lo cual puede suceder con las especies dominantes de la captura de descarte en Veracruz.

Sin embargo, para sustentar este tipo de inferencias es necesario generar series de tiempo sobre la composición de las capturas comerciales para detectar tendencias de variación de las comunidades de peces demersales. Así mismo, es preciso conocer las tallas máximas de las especies, su ciclo de vida, resiliencia y estimaciones de abundancias independientes de la captura comercial, para detectar el impacto funcional sobre las comunidades de peces.

Además del componente eminentemente demersal en el descarte, resalta la presencia de cuatro especies pelágicas costeras en el descarte, con abundancia y frecuencia relativa menor en la FAC y por ende con riesgo de impacto bajo: Anchoa hepsetus, Sardinella aurita, Decapterus punctatus y Scomberomorus maculatus. Estas especies se pueden considerar como capturas incidentales fortuitas ya que son de hábitos pelágicos y formadoras de grandes cardúmenes (Froese \& Pauly 2016), y son atrapadas durante el calado o cobrado del equipo.

Además de las alteraciones en las funciones ecológicas de la comunidad demersal, se pueden percibir también impactos sobre aspectos socioeconómicos (Duarte 2008), principalmente por la captura masiva de tallas de pre-reclutas y pre-adultos de especies con importancia económica para otras pesquerías dirigidas, tal y como sucede con la captura de especies de Lutjanidae, Priacanthidae, Malacanthidae, Trichiuridae, Balistidae y Tetraodontidae, registradas en el presente estudio. Este fenómeno representa una fuente de impacto negativo al proceso de reclutamiento al stock pesquero, del cual se abastece la pesquería artesanal de la zona (Jiménez-Badillo \& Castro-Gaspar 2007) y puede considerarse como una sobrepesca de especies comerciales y no comerciales (Vianna \& Verani 2002, Jeffers et al. 2008) durante el período de muestreo aquí descrito.

Dado el impacto primordial y recurrente sobre tallas pequeñas observado también por Duarte et al. (2006), la pesquería de arrastre en zonas tropicales tiene un mayor efecto en el ámbito ecológico que en el comercial (Hall 1999). El reto a resolver, a corto plazo, es que de las especies que presentan alta dominancia y alto valor de importancia en este estudio, se cuente con la información referente a la talla de primera madurez sexual y su dinámica poblacional en general, ya que ésta es escasa y fragmentada. No obstante, se puede inferir el impacto a estas especies a través de las tallas máximas publicadas. De esta forma, la talla máxima $\left(\mathrm{L}_{\max }\right)$ registrada para Citharichthys macrops es de $20 \mathrm{~cm}$ (Pauly \& Froese 2016), contrastando con las tallas promedio de captura obtenidas en este estudio $(12,95 \pm 6,42 \mathrm{~cm})$. Synodus foetens, Cruz-Escalona et al. (2005) mencionan que la pesca de arrastre camaronero impacta a organismos jóvenes, como es el caso del presente estudio (LT promedio 14,05 $\pm 5,70 \mathrm{~cm}$ ), si se toma en cuenta que se reporta una $\mathrm{L}_{\max }$ de $26,2 \mathrm{~cm}$ (Jeffers et al. 2008). Lo mismo sucede para Diplectrum bivittatum que presentó tallas menores a 12,0 cm siendo que la $\mathrm{L}_{\text {max }}$ estimada es de $25,0 \mathrm{~cm}$ (Pauly \& Froese 2016). Para el caso de Rhomboplites aurorubens, Freitas et al. (2011) estimó que la talla de primera madurez sexual $\left(\mathrm{L}_{50}\right)$ se encuentra entre 15,3 a $17,5 \mathrm{~cm}$, siendo que los organismos más grandes capturados en el presente estudio se encuentran por debajo de este umbral (talla promedio de 10,84 $\pm 1,66 \mathrm{~cm})$. Upeneus parvus presentó tallas de descarte por debajo de los $12 \mathrm{~cm}$, es decir menos del $40 \%$ de 
la $\mathrm{L}_{\max }$, que de acuerdo a Pauly \& Froese (2016), es de 30,0 $\mathrm{cm}$. Por último, Scorpaena calcarata, la cual no presenta ningún tipo de uso potencial, presentó una talla promedio 8,69 $\pm 2,39 \mathrm{~cm}$, lo cual se encuentra por arriba del intervalo de talla reportada por Acero \& Navas (1998) (2,91 a 6,75 cm). De acuerdo a lo anterior, se debe considerar que las especies dominantes del descarte están siendo capturadas mayoritariamente antes de la talla de primera madurez sexual, y para algunas de ellas, también en su fracción adulta.

La importancia ecológica de la ictiofauna en los fondos marinos y su relevancia comercial, reside en su contribución al proceso de transporte de nutrientes y energía entre especies y ecosistemas adyacentes, a través de su integración en la red trófica (González-Gándara et al. 2012). Los cambios en la estructura de las comunidades y en sus patrones de distribución resultan en un agotamiento sistemático que puede ser ilustrado de diversas formas, siendo el nivel trófico de la captura ejemplo de lo anterior, lo que implica que las pesquerías tienden a capturar cada vez más especies de la parte inferior de la trama trófica (Rice 2000, Board 2002, Barnes \& Thomas 2005, Løkkeberg 2005, Pauly \& Palomares 2005), modificando la estructura de tallas y reduciendo la riqueza (Ball et al. 2000, Kaiser et al. 2002, Hiddink et al. 2006).

En este sentido, la evaluación del nivel trófico del descarte en la pesca de arrastre en Veracruz, a través de la aproximación obtenida por Fishbase (Froese \& Pauly 2016), se convierte en un aspecto importante a considerar para futuras evaluaciones sobre el impacto de la pesquería sobre la comunidad demersal. El nivel trófico de la captura obtenido en el presente estudio $(3,84$ a 4,17) es reflejo de la dominancia de especies con hábitos piscívoros, como lo son Synodus foetens y Scorpaena calcarata, sumado a especies de la familia Triglidae, Tetradontidae y Carangidae, especies anteriormente reportadas para la zona de estudio por Peláez-Rodríguez et al. (2005).

Debido a la escasa información sobre la captura de descarte en la región, el análisis de la ictiofauna del presente estudio es el punto de partida en la valoración del efecto de la pesca de arrastre sobre la comunidad demersal, caracterizado por la captura de tallas pequeñas de especies con importancia comercial y ecológica, nivel trófico alto, alta dominancia y baja riqueza de especies como lo descrito para el Caribe colombiano (Duarte et al. 2006).

Sumado a lo anterior, se debe de tomar en cuenta la influencia de los sistemas estuarinos en la zona costera del centro sur del estado de Veracruz, los cuales inducen cambios en las condiciones de turbidez y salinidad, siendo factores determinantes en las condiciones de vida de los organismos (Manjarrés et al. 2001). En este sentido, la estructura de la comunidad de peces demersales a lo largo de la zona donde se realiza la pesca de arrastre, está relacionada con el ciclo de vida de las especies y a las condiciones ambientales, determinadas por la descarga de los sistemas estuarinolagunares de Alvarado-Papaloapan (S3B) al norte y el río Coatzacoalcos (SB10) en el sur. La influencia de estos sistemas resulta en altos niveles de productividad (Morán et al. 2005, Abarca-Arenas et al. 2004) con el consecuente aumento de recursos disponibles en la plataforma continental, convirtiéndola en un área importante de alimentación para la ictiofauna demersal que forma parte de la FAC (Yáñez-Arancibia 1985, Peláez-Rodríguez et al. 2005).

Así mismo, los parámetros ecológicos de riqueza de especies y diversidad están relacionados con el perfil batimétrico del área de arrastre, el estrato de 22 a 46 m albergó el valor máximo de riqueza, en tanto que, el estrato de profundidad $>46 \mathrm{~m}$ fue el de máxima diversidad, lo que sugiere que el grado de impacto probable del arrastre camaronero, es menos evidente en los límites de la distribución batimétrica de volúmenes comerciales de camarón.

En este sentido, el análisis de los cuadrantes de pesca a través del uso del MDS (Fig. 5) permitió obtener una compresión más clara de la relación de la composición y la influencia de los sistemas lagunares así como de la profundidad a lo largo de la zona de arrastre camaronero y de cómo los valores de riqueza (número de especies) obtenidos afectó las asociaciones. El cuadrante $\mathrm{S} 1 \mathrm{C}$ que registró la riqueza más baja (10 especies), al norte del Puerto de Veracruz frente a la desembocadura del río La Antigua, siendo un área en la cual no se realizan arrastres regularmente, debido a que los fondos se caracterizan por tener una gran cantidad de restos de árboles y los rendimientos son muy bajos.

Otro de los agrupamientos aislados, se caracterizó por recibir la influencia directa de las descargas de los ríos Coatzacoalcos (cuadrante S10B) y Papaloapan (S3B), con una mayor presencia de especies estuarino-dependientes y con los valores más altos de riqueza.

El ensamblaje más grande identificado corresponde a los cuadrantes de muestreo que se encuentran al sureste del sistema lagunar de Alvarado hasta las inmediaciones de la laguna del Ostión (S4C, S5C, S6C, S7C, S8B, S9B y S9C), la mayoría de ellos correspondieron al intervalo de profundidad $\mathrm{C}$ y la riqueza de especies registró valores que van de 12 a 14 .

El último agrupamiento identificado se caracteriza por valores de riqueza de 16 a 18 especies, siendo el segundo agrupamiento con mayor riqueza resultado, posiblemente debido a la influencia de los sistemas estuarinos localizados en la zona y está conformado por los cuadrantes ubicados en el intervalo de profundidad B, mayormente, abarcado desde el 
Puerto de Alvarado hasta las inmediaciones del puerto de Coatzacoalcos.

Si bien la posición de los agrupamientos pueden evidenciar las diferencias en la composición de especies resultado de la influencia de los sistemas estuarinos dentro de una temporada climática, sería de gran valor información continua que permita completar el ciclo anual y con ello identificar patrones estacionales, geográficos, batimétricos y sus interacciones, y así comprobar el posible uso de la zona costera y plataforma continental por la comunidad íctica demersal, a la par de documentar los cambios provocados por la actividad de arrastre camaronero.

El estudio sobre la composición y estructura de la fauna de descarte es relevante, más aún cuando se trata de evaluar el impacto de esta pesquería sobre el hábitat, las comunidades demersales y sus interacciones, como parte de la ordenación pesquera bajo el marco del enfoque ecosistémico de las pesquerías (SOFIA 2008, Herrera-Valdivia et al. 2016). Debido a que la evaluación de la captura y dinámica del descarte es uno de los principales problemas asociados al arrastre camaronero, la sistematización de largo plazo de este tipo de estudios cobra relevancia si se toma en cuenta que la pesquería de camarón en el Golfo de México genera alrededor de 19.000 ton de descartes (López-Martínez et al. 2012).

La pérdida de la diversidad como consecuencia de la remoción de especies por el arrastre camaronero en mares tropicales es un tema relevante y de actualidad a nivel internacional (Eayrs 2007), por lo tanto el estudio de la composición del descarte, es fundamental para cimentar la evaluación de las consecuencias y escenarios que conlleva la remoción de especies por los arrastres camaroneros en la costa veracruzana, y al mismo tiempo debe convertirse en una herramienta básica para el manejo de la pesquería bajo el enfoque ecosistémico para el estado mexicano. Finalmente, la aproximación metodológica, analítica y la información presentada puede servir como base para monitorear los cambios futuros que resulten de la reducción o aumento del esfuerzo pesquero, las modificaciones previsibles de las redes de arrastre en la pesquería del camarón, así como evidenciar cualquier otro co-factor de variabilidad como el forzamiento ambiental.

\section{Agradecimientos}

Los autores agradecen el apoyo otorgado por el Centro Regional de Investigación Acuícola y Pesquera - Veracruz-INAPESCA en la obtención de las muestras. A la flota camaronera del puerto deAlvarado, Veracruz por permitirnos participar en las labores de pesca de camarón durante la temporada 2013. Al Consejo Nacional de Ciencia y Tecnología (CONACYT) por el apoyo a través del otorgamiento de Beca Nacional CVU 516004 para estudios de doctorado en el Programa de Doctorado en Ecología y Pesquerías. Así mismo, al Instituto de Ciencias Marinas y Pesquerías de la Universidad Veracruzana por las facilidades en el uso de sus instalaciones para el procesamiento de muestras y análisis de la información. Y por último, a la editora y revisores de la Revista de Biología Marina y Oceanografía por sus acertadas sugerencias y correcciones al manuscrito.

\section{LITERATURA CITADA}

Abarca-Arenas LG, J Franco-López, R Chávez-López, D Arceo-Carranza \& A Morán-Silva. 2004. Trophic analysis of the fish community taken as bycatch of shrimp trawls off the coast of Alvarado, Mexico; Análisis trófico de la comunidad de peces provenientes de la pesca incidental camaronera de la costa de Alvarado, México. 55 $5^{\text {th }}$ Gulf and Caribbean Fisheries Institute, GCFI 55: 384-394.

Abarca-Arenas LG, J Franco-López, MS Peterson, NJ Brown-Peterson \& E Valero-Pacheco. 2007. Sociometric analysis of the role of penaeids in the continental shelf food web off Veracruz, Mexico based on by-catch. Fisheries Research 87: 46-57.

Abarca-Arenas LG, J Franco-López, C González-Gándara \& G Silva-López. 2012. Los peces de la costa veracruzana: relación especies-área y conectividad entre sitios. Investigación Ictiológica en México, Temas Selectos en Honor al Dr. José Luis Castro Aguirre, Universidad Nacional Autónoma de México, México 304(11): 127-158.

Acero A \& GR Navas. 1998. Notes on the fish family Scorpaenidae (Pisces: Scorpaeniformes) in the continental Colombian Caribbean, including a new record. Oceanographic Literature Review 7(45): 1184-1185.

Alverson DL, MH Freeberg, JG Pope \& SA Murawski. 1994. A global assessment of fisheries bycatch and discards FAO Fisheries Technical Paper 339: 1-233.

Báez RC \& MP Balsinde. 2008. Aprovechamiento de la fauna de acompañamiento (FAC) del camarón en Costa Rica. Revista Cubana de Investigaciones Pesqueras 25(1): 75-79.

Ball B, B Munday \& I Tuck. 2000. Effects of otter trawling on the benthos and environment in muddy sediments. In: Kaiser MJ \& J de Groot (eds). The effects of fishing on non-target species and habitats, pp. 69-82. Blackwell Science, Oxford.

Barnes PW \& JP Thomas. 2005. Benthic habitats and the effects of fishing. American Fisheries Society Symposium 41: 1-890.

Board OS. 2002. Effects of trawling and dredging on seafloor habitat, 125 pp. The National Academies Press, Washington.

Bouchon-Navaro Y, C Bouchon, D Kopp \& M Louis. 2006. Weight-length relationships for 50 fish species collected in seagrass beds of the Lesser Antilles. Journal of Applied Ichthyology 22(4): 322-324. 
Carpenter SR. 2002. Ecological futures: building an ecology of the long now. Ecology 83(8): 2069-2083.

Casteló-Báez R \& MP Balsinde. 2008. Aprovechamiento de la fauna de acompañamiento (FAC) del camarón en Costa Rica. Revista Cubana de Investigaciones Pesqueras 25(1): 75-91.

Castro-Aguirre JL. 1999. Ictiofauna estuarino-lagunar y vicaria de México, 635 pp. Editorial Limusa, México.

Charles AT. 2001. Sustainable fishery systems. Fish and Aquatic Resources Series 5: 1-367, Blackwell Science, Oxford.

Chávez-López R, J Franco-López, A Morán-Silva \& MT O'Connell. 2005. Long-term fish assemblage dynamics of the Alvarado Lagoon Estuary, Veracruz, Mexico. Gulf and Caribbean Research 17(1): 145-156.

Clucas I. 1998. Bycatch-is it a bonus from the sea. Infofish International 3: 24-26.

CONAPESCA. 2013. Anuario estadístico de acuacultura y pesca 2013. Secretaría de Agricultura, Ganadería, Desarrollo Rural, Pesca y Alimentación, México. <http:// www.conapesca.sagarpa.gob.mx/wb/cona/anuario_2013>

Cruz-Escalona VH, MS Peterson, L Campos-Dávila \& M Zetina-Rejón. 2005. Feeding habits and trophic morphology of inshore lizardfish (Synodus foetens) on the central continental shelf off Veracruz, Gulf of Mexico. Journal of Applied Ichthyology 21(6): 525-530.

Duarte LO. 2008. Coastal ecosystem status in bottom-up vs. top. In: Datta R (ed). Coastal ecosystems. hazards, management and rehabilitation, pp. 249-260. NAM S\&T Centre, Delhi.

Duarte LO, P Gómez-Canchong, LM Manjarrés, CB García, FD Escobar, J Altamar \& F Cuello. 2006. Variabilidad circadiana de la tasa de captura y la estructura de tallas en camarones e ictiofauna acompañante en la pesquería de arrastre del Mar Caribe de Colombia. Investigaciones Marinas 34(1): 23-42.

Duarte LO, L Manjarrés \& F Escobar. 2010. Bottom trawl bycatch assessment of the shrimp fishery in the Caribbean Sea off Colombia. In: Proceedings of the Gulf and Caribbean Fisheries Institute 62: 114-119. Gulf and Caribbean Fisheries Institute, c/o Harbor Branch Oceanographic Institution, Fort Pierce.

Eayrs S. 2007. Guía para reducir la captura de fauna incidental (bycatch) en las pesquerías por arrastre de camarón tropical. Edición revisada, 108 pp. FAO, Roma.

FAO. 2003. La ordenación pesquera. 2. El enfoque de ecosistemas en la pesca. Orientaciones Técnicas para la Pesca Responsable 4, Supl. 2: 1-133. FAO, Roma.

FAO. 2012. Departamento de Pesca y Agricultura. La pesca de camarón, a examen. FAO, Roma. <http://www.fao.org/ news/story/es/item/10164/icode/. Agosto de 2016>
Freitas MO, RL de Moura, RB Francini-Filho \& CV Minte-Vera. 2011. Spawning patterns of commercially important reef fish (Lutjanidae and Serranidae) in the tropical western South Atlantic. Scientia Marina 75(1): 135-146.

Froese R \& D Pauly. 2016. FishBase. < www.fishbase.org>

García E. 1982. Modificaciones al sistema de clasificación climática de Köppen: para adaptarlo a las condiciones de la República Mexicana. Serie Libros 6: 1-246, Instituto de Geografía, Universidad Nacional Autónoma de México. México.

Gillett R. 2008. Global study of shrimp fisheries. FAO Fisheries Technical Paper 475: 1-331.

Giménez-Hurtado E, A Pérez-Marrero, G DelgadoMiranda, HA Domínguez \& V Villafuerte-Delgado. 2016. Comportamiento de la fauna acompañante en la pesca de camarón rosado (Farfantepenaeus notialis) en la Plataforma suroriental de Cuba. REDVET. Revista Electrónica de Veterinaria 17(11): 1-22.

González-Gándara C. 2011. La zona marina. En: Cruz-Angón A (ed). La biodiversidad en Veracruz: Estudio de Estado, pp. 293-300. Comisión Nacional para el Conocimiento y Uso de la Biodiversidad (CONABIO), Gobierno del Estado de Veracruz, Universidad Veracruzana, Instituto de Ecología, A. C., México, D. F.

Gónzalez-Gándara C, VDL Francisco \& J Salas. 2012. Lista de los peces de Tuxpan, Veracruz, México. Revista Científica UDO Agrícola 12(3): 675-689.

Grainger RJR \& SM García. 1996. Chronicles of marine fishery landings (1950-1994): trend analysis and fisheries potential, $51 \mathrm{pp}$. FAO, Rome.

Grande VJ \& LM Díaz. 1981. Situación actual y perspectivas de utilización de la fauna de acompañamiento del camarón en México. Ciencia Pesquera 1(2): 43-55.

Hall SJ. 1999. The effects of fishing on marine ecosystems and communities, $274 \mathrm{pp}$. Blackwell Science, Oxford.

Hammer O \& D Harper. 2003. PAST: Paleontological statistics software package for education and data analysis, Version 1.15. <http://palaeo-electronica.org/2001_1/past/issue 1 01.htm>

Herrera-Valdivia E, J López-Martínez \& S CastilloVargasmachuca. 2015. Estrés en la comunidad íctica en la pesca de arrastre del camarón en el norte del Golfo de California. Revista de Biología Tropical 63(3): 741-754.

Herrera-Valdivia E, J López-Martínez, S CastilloVargasmachuca \& AR García-Juárez. 2016. Diversidad taxonómica y funcional en la comunidad de peces de la pesca de arrastre de camarón en el norte del Golfo de California, México. Revista de Biología Tropical 64(2): 587-602.

Hiddink JG, T Hutton, S Jennings \& MJ Kaiser. 2006. Predicting the effects of area closures and fishing effort restrictions on the production, biomass, and species richness of benthic invertebrate communities. ICES Journal of Marine Science: Journal du Conseil 63(5): 822-830.

Hoese HD \& RH Moore. 1998. Fishes of the Gulf of Mexico, 422 pp. Texas A \&M University Press, College Station. 
Jeffers SA, WF Patterson III \& JH Cowan Jr. 2008. Habitat and bycatch effects on population parameters of inshore lizardfish (Synodus foetens) in the north central Gulf of Mexico. Fishery Bulletin 106(4): 417-426.

Jennings S \& MJ Kaiser. 1998. The effects of fishing on marine ecosystems. Advances in Marine Biology 34: 201352.

Jennings S \& JD Reynolds. 2000. Impacts of fishing on diversity: from pattern to process. Effects of fishing on nontarget species and habitats, pp. 235-250. Blackwell Science, Oxford.

Jiménez R. 1979. Características hidrográficas de la Vertiente del Golfo de México en el Estado de Veracruz. Investigaciones Geográficas 9: 117-156.

Jiménez-Badillo ML \& L Castro-Gaspar. 2007. Pesca artesanal en el Parque Nacional Sistema Arrecifal Veracruzano, México. En: Granados-Barba A, LG AbarcaArenas \& JM Vargas-Hernández (eds). Investigaciones científicas en el Sistema Arrecifal Veracruzano, pp. 221-240. Universidad Autónoma de Campeche, Campeche.

Kaiser MJ, JS Collie, SJ Hall, S Jennings \& IR Poiner. 2002. Modification of marine habitats by trawling activities: prognosis and solutions. Fish and Fisheries 3(2): 114-136.

Kruskal WH \& WA Wallis. 1952. Use of ranks in one-criterion variance analysis. Journal of the American Statistical Association 47: 583-621.

Lara-Domínguez AL, J Franco-López, C Bedia-Sánchez, LG Abarca-Arenas, S Díaz-Ruiz, A Aguirre-León, C González-Gándara \& M Castillo-Rivera. 2011. Diversidad de peces en los ambientes costeros y plataforma continental. En: Cruz-Angón A (ed). La biodiversidad en Veracruz: Estudio del Estado, pp. 825-836. CONABIO, Instituto de Ecología, A. C., México, D. F.

Lohrenz SE, DA Wiesenburg, RA Arnone \& X Chen. 1999. What controls primary production in the Gulf of Mexico? In: Kumpf H, K Sherman \& K Steidinger (eds). The Gulf of Mexico Large Marine Ecosystem - Assessment, Sustainability and Management, pp. 151-170. Blackwell Science, Malden.

López-Martínez J, E Herrera-Valdivia, J RodríguezRomero \& S Hernández-Vázquez. 2010. Peces de la fauna de acompañamiento en la pesca industrial de camarón en el Golfo de California, México. Revista de Biología Tropical 58(3): 925-942.

López-Martínez J, E Herrera-Valdivia, N HernándezSaavedra, E Serviere-Zaragoza, J Rodríguez-Romero, CH Rábago-Quiroz, G Padilla-Arredondo, $S$ BurrolaSánchez, D Urias-Laborín, R Morales-Azpeitia, S Pedrín-Aviles, LF Enríquez-Ocaña, MO NevárezMartínez, A Acevedo-Cervantes, E MoralesBojórquez, M del R López-Tapia \& J Padilla-Serrato. 2012a. Efectos de la pesca de arrastre del camarón en el Golfo de California. Síntesis de las investigaciones desarrolladas por el Centro de Investigaciones Biológicas del Noroeste SC. En: López-Martínez J \& E MoralesBojórquez (eds). Efectos de la pesca de arrastre en el Golfo de California, pp. 15-26. Centro de Investigaciones Biológicas del Noroeste, SC y Fundación Produce Sonora, México.
López-Martínez J, S Hernández-Vázquez, R MoralesAzpeitia, MO Nevárez-Martínez, C Cervantes-Valle \& J Padilla-Serrato. 2012b. Variación de la relación camarón: fauna de acompañamiento en la pesquería de camarón industrial del Golfo de California. En: LópezMartínez J \& E Morales-Bojórquez (eds). Efectos de la pesca de arrastre en el Golfo de California, pp. 27-47. Centro de Investigaciones Biológicas del Noroeste, SC y Fundación Produce Sonora, México.

Løkkeberg S. 2005. Impacts of trawling and scallop dredging on benthic communities. FAO Fisheries Technical Paper 472: $1-58$.

Madrid-Vera J, F Amezcua \& E Morales-Bojórquez. 2007. An assessment approach to estimate biomass of fish communities from bycatch data in a tropical shrimp-trawl fishery. Fisheries Research 83(1): 81-89.

Magurran AE \& BJ McGill. 2011. Biological diversity: frontiers in measurement and assessment, $345 \mathrm{pp}$. Oxford, New York.

Manjarrés L, C García \& A Acero. 2001. Caracterización ecológica de las asociaciones de peces demersales del Caribe colombiano norte, con énfasis en los pargos (Lutjanidae). Boletín de Investigaciones Marinas y Costeras 30: 77-107.

Matteucci SD \& A Colma. 1982. Metodología para el estudio de la vegetación, 72 pp. Secretaría General de la Organización de Estados Americanos, Programa Regional de Desarrollo Científico y Tecnológico, Washington.

Monk MH, JE Powers \& EN Brooks. 2015. Spatial patterns in species assemblages associated with the northwestern Gulf of Mexico shrimp trawl fishery. Marine Ecology Progress Series 519: 1-12.

Morán-Silva A, LA Martínez-Franco, R Chávez-López, J Franco-López, CM Bedia-Sánchez, F Contreras, F Gutiérrez-Mendieta, NJ Brown-Peterson \& MS Peterson. 2005. Seasonal and spatial patterns in salinity, nutrients, and chlorophyll $a$ in the Alvarado Lagoon System, Veracruz, Mexico. Gulf and Caribbean Research 17(1): 133143.

Page LM, H Espinosa-Pérez, LT Lindley, CR Gilbert, RN Lea, NE Mandrake, NE Mayden \& JS Nelson. 2013. Common and scientific names of fishes from the United States, Canada, and México. American Fisheries Society, Special Publication 34: 1-243.

Páramo J, L Guillot-Illidge, S Benavides, A Rodriguez \& C Sánchez-Ramírez. 2009. Aspectos poblacionales y ecológicos de peces demersales de la zona norte del Caribe colombiano en relación con el hábitat: una herramienta para identificar Áreas Marinas Protegidas (AMPs) para el manejo pesquero. Caldasia 31(1): 123-144.

Pauly D \& ML Palomares. 2005. Fishing down marine food web: it is far more pervasive than we thought. Bulletin of Marine Science 76(2): 197-212.

Peláez-Rodríguez E, J Franco-López, WA Matamoros, R Chávez-López \& NJ Brown-Peterson. 2005. Trophic relationships of demersal fishes in the shrimping zone off Alvarado Lagoon, Veracruz, Mexico. Gulf and Caribbean Research 17(1): 157-167. 
Rábago-Quiroz H, J López-Martínez, JE Valdez-Holguín \& MO Nevárez-Martínez. 2011. Distribución latitudinal y batimétrica de las especies más abundantes y frecuentes en la fauna acompañante del camarón del Golfo de California, México. Revista de Biología Tropical 59(1): 255-267.

Rice JC. 2000. Evaluating fishery impacts using metrics of community structure. ICES Journal of Marine Science 57(3): 682-688.

Rodríguez-Romero J, DS Palacios-Salgado, J LópezMartínez, S Hernández-Vázquez \& G Ponce-Díaz. 2008. Composición taxonómica y relaciones zoogeográficas de los peces demersales de la costa occidental de Baja California Sur, México. Revista de Biología Tropical 56(4): 1765-1783.

SAGARPA. 2011. Instituto Nacional de Pesca. Dictamen Técnico. Fundamento técnico para el establecimiento de vedas para la pesca de camarón en el Golfo de México y Mar Caribe (2011), 44 pp. <www.inapesca.gob.mx/portal/ component/docman/doc_download/166-fundamento-tecnicopara-el-establecimiento-de-vedas-para-la-pesca-decamaron-en-el-golfo-de-mexico-y-mar-caribe>

SAGARPA. 2013. Instituto Nacional de Pesca. Dictamen Técnico. Fundamento técnico para el establecimiento de vedas para la pesca de camarón en el Golfo de México y Mar Caribe (2013), 41 pp. <http://inapesca.gob.mx/portal/ documentos/dictamenes/DICTAMENCAMARON-Veda2014.pdf>

SAGARPA-INAPESCA. 2010. Dictamen Técnico. Estimación de la fecha óptima para la apertura de la temporada 20102011 de la pesquería de camarón café (Farfantepeneaus aztecus) en las costas de Tamaulipas y Veracruz. Informe Técnico, Instituto Nacional de Pesca, $10 \mathrm{pp}$. <www.inapesca.gob.mx/portal/publicaciones/dictamenes/ doc_download/176-estimacion-de-la-fecha-optima-para-laapertura-de-la-temporada-20122013-de-la-pesqueria-decamaron>

Sampieri RH, CF Collado, PB Lucio \& MDLLC Pérez. 1998. Metodología de la investigación 1: 1-656. McGrawHill, México.

Scott-Denton E, PF Cryer, MR Duffy, JP Gocke, MR Harrelson, DL Kinsella \& JA Williams. 2012. Characterization of the US Gulf of Mexico and South Atlantic penaeid and rock shrimp fisheries based on observer data. Marine Fisheries Review 74(4): 1-27.

Slavin JW. 1981. Utilization of the shrimp by-catch. In: Fish bycatch - bonus from the sea. Report of a Technical Consultation on Shrimp Bycatch Utilization, 27-30 October 1981, Georgetown, Guyana, FAO/IDRC Ottawa (IDRC198e): 21-28.
SOFIA. 2008. El estado mundial de la pesca y la acuacultura 2008 (SOFIA). FAO, Roma. <http://www.fao.org/3/ai0250s.pdf>

Sokal RR \& FJ Rohlf. 2003. Biometry, 887 pp, W.H. Freeman and Company, New York.

Sparre P \& SC Venema. 1995. Introducción a la evaluación de stocks de peces tropicales. Parte 1-Manual. FAO Documento Técnico de Pesca 306/1 Rev. 1: 1-421.

Valdés E, V Villafuerte, H Domínguez \& A Pérez. 2010. Estructura de tallas de la ictiofauna en la pesquería de camarón en el Golfo de Ana María, plataforma suroriental de Cuba. Centro de Investigaciones Pesqueras. <http:// www.oceandocs.org/bitstream/handle/1834/3835/ Estructura\% 20 tallas \% 20 ictiofauna\% 202002 2010[1].pdf?sequence $=1>$

Valdés E, V Villafuerte, H Domínguez \& A Pérez. 2011. Variabilidad temporal de la fauna acompañante del camarón Farfantepenaeus notialis en el Golfo de Ana María, Cuba. Revista Cubana de Investigaciones Pesqueras 28(2): 1-7.

Vianna M \& JR Verani. 2002. Biologia populacional de Orthopristis ruber (Teleostei, Haemulidae) espécie acompanhante da pesca de arrasto do camarão-rosa, no sudeste brasileiro. Atlântica 23(1): 27-36.

Vianna M \& T Almeida. 2005. Bony fish bycatch in the southern Brazil pink shrimp (Farfantepenaeus brasiliensis and $F$. paulensis) fishery. Brazilian Archives of Biology and Technology 48(4): 611-623.

Wakida-Kusunoki AT, R Solana-Sansores, ME SandovalQuintero, G Núñez-Márquez, J Uribe-Martínez, A González-Cruz \& M Medellín-Ávila. 2006. Camarón del Golfo de México y Mar Caribe. En: Sustentabilidad y pesca responsable en México, evaluación y manejo, pp. 427-476. Instituto Nacional de la Pesca. Secretaría de Agricultura, Ganadería, Desarrollo Rural, Pesca y Alimentación, México.

Wakida-Kusunoki AT, I Becerra-de la Rosa, A GonzálezCruz \& LE Amador-del Ángel. 2013. Distribución y abundancia de la fauna acompañante del camarón en la costa de Tamaulipas, México (veda del 2005). Universidad y Ciencia 29(1): 75-86.

Wells RD, JH Cowan Jr \& WF Patterson III. 2008. Habitat use and the effect of shrimp trawling on fish and invertebrate communities over the northern Gulf of Mexico continental shelf. ICES Journal of Marine Science 65(9): 1610-1619.

Yáñez-Arancibia A. 1986. Los peces demersales de la plataforma continental del Sur del Golfo de México. 1. Caracterización ambiental, ecología y evolución de las especies, poblaciones y comunidades. Publicaciones Especiales Instituto de Ciencias del Mar y Limnología 9: 1229.

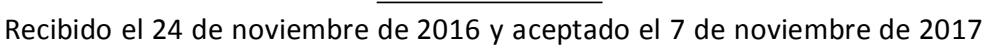

Editor: Claudia Bustos D. 